F. W. J. Hazebroek

R. M. M. Smeets

A. P. Bos

C. Ouwens

D. Tibboel

J. C. Molenaar

\section{Staff attitudes towards continuation of life-support in newborns with major congenital anomalies}

Received: 1 October 1995

Accepted: 22 February 1996

F. W. J. Hazebroek $(\bowtie) \cdot$ R. M. M. Smeets C. Ouwens - D. Tibboel - J. C. Molenaar Department of Paediatric Surgery Sophia Children's Hospital

Dr. Molewaterplein 60, 3015 GJ Rotterdam, The Netherlands Tel.: (31) (10) 4636783

Fax: (31) (10) 4636802

A. P. Bos

Department of Paediatrics,

Paediatric Intensive Care Unit, Beatrix Clinic/

Academic Hospital Groningen,

Groningen, The Netherlands

\begin{abstract}
This study was conducted to gain insight into the attitudes of medical staff towards life-support of newborns with life-threatening problems, seen against the background of these children's expected morbidity and quality of life. The opinions about the mode of life-support were determined by questonnaires and the demographic characteristics of the respondents were noted. Each patient's risk of mortality was scored by means of the standardized Paediatric Risk of Mortality Score (PRISM). Attitudes towards support were unanimous for 39 of the $46 \mathrm{pa}$ tients. For the other 7 patients at least one of the respondents preferred a different support mode than that given at the time. The attitudes
\end{abstract}

were influenced by the patient's risk of mortality.

Conclusion Decisions about lifesupport of newboms with lifethreatening problems should include all the disciplines involved in patient care and should be made at an early stage.

Key words Ethics, medical . Intensive care, neonatal $\cdot$ Lifesupport care - Attitude of health personnel $\cdot$ Quality of life

Abbreviations PRISM paediatric risk of mortality $\cdot I C U$ intensive care unit $\cdot C P R$ cardiopulmonary resuscitation - SICS senior intensive care specialist

\section{Introduction}

Recent advances in paediatric intensive care give better chances of survival in newborns with major congenital anomalies or infants developing life-threatening diseases. A number of these children are at risk of residual physical or mental handicaps following intensive care treatment [8]. Medical staff responsible for the management of these children have to balance, in close consultation with the children's parents, whether to start or continue life-support measures with the expected quality of life $[1,5,11]$.

In most intensive care units (ICU) decisions about lifesupport are made only when a critical clinical situation arises [6]. However, in our opinion such decisions should be made before a crisis occurs because the need for emer- gency measures often arises unexpectedly. In most Dutch Paediatric ICUs these decisions are made by the attending physician, in consultation with the patient's parents [5, 10]. Since ICU nurses and physiotherapists provide the greater part of primary care, they contribute markedly to the promptness and intensity of supportive care for highrisk infants. Indirectly they have an important role in lifesupport decision making. They are usually the first ones present when a crisis occurs and cardiopulmonary resuscitation (CPR) is required.

We conducted a prospective study designed to evaluate the attitudes of all disciplines in a paediatric surgical ICU towards life-support management of the patients they were taking care of. We also wanted to define parameters that may influence these attitudes. 


\section{Methods}

This study was conducted at the paediatric surgical ICU of the Sophia Children's Hospital, a tertiary referral centre. This unit comprises seven intensive care and seven high care beds for patients of all surgical subspecialities, and yearly admits some 500 patients, including approximately 100 neonates with major congenital anomalies. The Institutional Review Board granted study approval and waived the informed consent requirement.

In our unit a protocol provides guidelines for the treatment team through the decision-making process and around continuing or foregoing life-support of a child (Table 1). During 3 months the attending physician completed a form for each patient on the 1st day of admission to one of the seven intensive care beds, stating the patient's diagnosis and age at time of admission. Each patient's mortality risk was then assessed three times a week by means of the Paediatric Risk of Mortality (PRISM) score. This scoring system quantifies severity of illness based on 14 routinely measured clinical and laboratory parameters of physiological instability. For each parameter, an abnormal range with number of points is assigned, reflecting is weighted contribution to overall severity of illness. The points are summed to obtain the total PRISM score. All the PRISM score variables directly reflect their contribution to the mortality risk $[4,9]$.

On every day that PRISM scores were assessed, a questionnaire was distributed to the physicians, nurses, and others involved, such as paedagogical therapists, the chaplain, and physiotherapists. These questionnaires were kept anonymous. Apart from demographic characteristics such as profession, age, gender, religious backround, and having children of their own, the respondents were asked for their attitude towards life-support management for the patient they were taking care of. The response was presented as a multiple choice, offering four options: (1) "full support, including CPR"; (2) "full support, excluding CPR"; (3) "modified support, excluding CPR" and (4) "no opinion". "Modified support" implied support directed towards basic comfort; forms of care that would produce discomfort or would not offer a prospect of meaningful recovery were withheld or withdrawn.

Student's $t$-test was used to compare the mean values of patient-bound variables (age, duration of admission, PRISM score) and respondent-bound variables (age, gender, profession, religion)

Table 1 The stages of the decision-making process on forgoing life-support treatment

Before death discussions between

1. Responsible Senior Intensive Care Specialist (SICS) and other involved medical and surgical staff members

2. SICS and nurses

3. SICS and nurses and parents "bad news talk"

4. Feedback to other involved staff members

5. (Repetition of "bad news talk" with parents)

6. SICS with nurses and parents-discussion about circumstances of death such as presence of parents, child in arms of parents

7. SICS with nurses - discussion about actual dying process

After death discussions between

1. SICS and nursing team-evaluation of dying process, feedback and evaluation

2. Treatment team-discussion, of complete medical history 1 week after death of child

3. On the initiative of the parents contact between SICS and parents-review medical history (including autopsy results), questions about future risk of recurrence, prenatal diagnosis, etc.
Table 2 Primary diagnosis on admission

\begin{tabular}{lr}
\hline Congenital anomalies & \\
Multiple congenital anomalies & $\mathrm{a}$ \\
Airway, lung anomaly & 2 \\
Congenital diaphragmatic hernia & 7 \\
Congenital heart disease & 2 \\
Digestive tract anomaly & 9 \\
Abdominal wall defect & 5 \\
Prune-belly syndrome & 1 \\
Cloacal extrophy & 2 \\
Epidermolysis bullosa & 1 \\
Other diseases & \\
Necrotizing enterocolitis & 5 \\
Brain tumour2 & \\
Total & 46 \\
\hline
\end{tabular}

${ }^{a}$ Multiple congenital anomalies were defined as two or more lifethreatening congenital disorders

in the group about whom attitudes were unanimous, and in the group about whom the attitudes towards management differed.

\section{Results}

During the study period 46 patients were admitted to one of the seven ICU beds. Two beds were continuously occupied by chronic ventilator-dependent patients who were excluded from the analysis. Nearly all patients were neonates with major congenital anomalies, the nature of which is detailed in Table 2.

Two hundred and ninety-eight questionnaires were filled in anonymously by ICU nurses, physicians, physiotherapists, child-life specialists, and hospital chaplains. Only in 5 questonnaires respondents (two ICU nurses, two physicians, one chaplain) opted for "no opinion" as concerning their attitude towards preferred life-support, leaving 293 completed questionnaires about the support of the 46 patients. All respondents unanimously opted for a "full support, including CPR" for 37 patients, reflecting the actual management. For two neonates, one with severe epidermolysis bullosa, the other with a hypoplastic left ventricle, an unanimous "modified support, excluding CPR " was indicated, as was the actual management.

With regard to the remaining 7 patients there was no unanimity in attitudes towards life-support. In this group the actual management and the preferred management of at least one of the respondents differed. Table 3 shows the chosen attitudes towards the support of these 7 patients. Fifteen questionnaires, completed by 13 different respondents, showed choices about life-support different from the actual management. In two cases the respondent felt that actual life-support was insufficient; in the other cases respondents indicated that CPR should be withheld or the patient's support should be reduced, whereas the current management included CPR or full support, respectively. 
Table 3 Actual management and chosen attitudes towards life-support of the patients about whom no unanimity was expressed. ( $F S$ full support, including CPR; FS/no CPR full support, excluding CPR; $M S$ modified support aimed at comfort, excluding CPR)

\begin{tabular}{lllllll}
\hline $\begin{array}{l}\text { Actual } \\
\text { management }\end{array}$ & $\begin{array}{l}\text { No. of } \\
\text { patients }\end{array}$ & \multicolumn{2}{l}{$\begin{array}{l}\text { Total No. of respondents } \\
\text { (No. of questionnaires) }\end{array}$} & \multicolumn{3}{l}{$\begin{array}{l}\text { Total No. of respondents with different } \\
\text { attitudes (No. of questionnaires) }\end{array}$} \\
\cline { 4 - 7 } \cline { 3 - 6 } & 5 & $27(54)$ & FS & FS/no CPR & MS \\
\hline FS & 1 & $6(7)$ & $1(1)$ & 7 & $(9)$ & $1(1)$ \\
FS/no CPR & 1 & $2(2)$ & $0(0)$ & 1 & $(1)$ & $3(3)$ \\
Total & 7 & $35(63)$ & $1(1)$ & $8(10)$ & $4(4)$ \\
\hline
\end{tabular}

The group of respondents with different attitudes consisted of seven ICU nurses, three physicians, one physiotherapist, one child-life specialist and one chaplain.

Regarding patient-bound variables, the mean ( \pm SE) PRISM score of the seven children responsible for diverging attitudes was $9.6 \pm 1.9$, which was significantly higher than the score of the children about whom the respondents' choice was unanimous: $4.1 \pm 0.3(P<0.05)$. There were no significant differences in age, duration of admission, or incidence of congenital anomalies between the two groups.

If we consider the 293 completed questionnaires, the risk of mortality turned out to be the only patient-bound variable correlating with the choice of life-support. The mean $( \pm$ SE) PRISM score of the patients for whom "full support, including CPR" was chosen, was significantly lower $(4.0 \pm 0.3)$ than the score of the patients for whom "full support, no CPR" was chosen $(10.1 \pm 2.4)$ and that of the patients for whom "modified support, no CPR" was preferred $(8.5 \pm 2.4)$. Other patient variables did not play a significant role in the choise of life-support.

No respondent-bound variable significantly influenced the attitude towards life-support.

\section{Discussion}

A comparable study, published over a decade ago, reporting staff attitudes towards life-support of the critically ill adult, showed a remarkable agreement between physicians and nurses on ethical issues [8]. No significant relationships between attitude towards life-support and religious preference, presence of children in their own family, or gender in either the physician or the nursing group were shown. In contrast to our study, these data were obtained by means of questionnaires about imaginary patients in hypothetical situations. Since only a low positive correlation between nurses' moral judgement about hypothetical general dilemmas and moral judgement about real-life nursing dilemmas has been described [2], our study concerned patients whom the respondents were actually taking care of. Thus we tried to take into account a possible influence of emotions and attachment to the patient on the respondent's attitudes towards life-support.

From another study conducted at our department, we learned that our protocol of staged decision making en- abled us to deal with our own feelings of grief because it guarantees feedback to all members of the treatment team [5]. In this study we found that opinions about life-support differed with regard to 7 of the 46 patients. Since physicians, nurses, and other therapists supply extended primary care frequently every day they are often confronted with patients for whom they feel support should be different. It is therefore necessary to discuss withholding and withdrawal of life-support at an early stage of treatment, involving all the disciplines in the decisionmaking process. Once a consensus is reached, do-not-resuscitate orders are written in the patients records before attitudes towards life-support will be changed. Sometimes more time is needed and new discussions have to be made within hours or days.

Did particular aspects influence the respondents in their attitude towards life-support of patients under their care? Children for whom no full life-support was preferred turned out to have, on the average, a higher risk of mortality. This may be explained by the essence of the PRISM score: it is constructed by scores of both the neurological and the cardiorespiratory condition [9]. The poor neurological state of the patient may imply a poor prognosis for attainment of personal awareness; a poor cardiorespiratory condition (e.g. severe bronchopulmonary dyplasia) influences the quality of life mainly physically [3]. An expected low probability of self-awareness and a poor quality of life of the patient could have played a role in the respondents' judgements. These approaches to ethical decision making in the neontal ICU have been suggested before [10]; our findings underline their relevance in practice.

In accordance with results of studies about imaginary patients we found no significant relationship between respondents' characteristics (profession, age, gender, religious background) and the attitude towards life-support. In this respect, studying attitudes towards life-support of real-life patients did not bring out new points of view [12]. We conclude that decisions about life-support of newborns with life-threatening problems should include all the ICU disciplines involved in patient care and should be made at an early stage. This offers the opportunity of detecting and discussing different attitudes. In this way, understanding between caring disciplines about the support of the children they are taking care of will be enlarged and misunderstanding, frustation and burn-out can be prevented [7]. 
We urge other ICU workers to adopt our approach in future studies because prospective surveys of attitudes and decision making in real-life dilemmas reveal much more than the usual reports of moral attitudes based on hypothetical situations.
Acknowledgements The authors thank A. P. Provoost, PhD, for the statistical data processing and J. Hagoort, MA, for editorial assistence.

\section{References}

1. Anonymous (1990) Consensus report on the ethics of foregoing life-sustaining treatments in the critically ill. Crit Care Med 18:1435-1439

2. Crisham P (1981) Measuring moral judgement in nursing dilemmas. Nurs Res 30: 104-110

3. Farrell PM, Fost NC (1989) Long-term mechanical ventilation in pediatric respiratory failure: medical and ethical considerations. Am Rev Respir Dis $140: S 36-S 40$

4. Gemke RJBJ, Bonsel GJ, Vught AJ van (1994) Effectiveness and efficiency of a Dutch pediatric intensive care unit: validity and application of the pediatric risk of mortality (PRISM) score. Crit Care Med 22: 1477-1484
5. Hazebroek FWJ, Tibboel D, Mourik M, Bos AP, Molenaar JC (1993) Withholding and withdrawal of life support from surgical neonates with life-threatening congenital anomalies. J Pediatr Surg 28: 1093-1097

6. Kyff J, Puri VK, Rajeha R, Ireland T (1987) Cardiopulmonary resuscitation in hospitalized patients: continuing problems of decision-making. Crit Care Med 15:41-43.11

7. Marshall RE, Kasman C (1980) Burnout in the neonatal intensive care unit. Pediatrics 65:1161-1165

8. Pollack MM, Wilkinson JD, Glass NC (1987) Long-stay paediatric intensive care patients: outcome and resource utilization. Pediatrics $8: 855-860$
9. Pollack MM, Ruttimann UE, Getson PR (1988) Pediatric risk of mortality (PRISM) score. Crit Care Med 16: 1110-1116

10. Sauer PJJ (1992) Ethical decisions in neonatal intensive care units: the Dutch experience. Pediatrics 90:729-731

11. Visser HKA, Aartsen HGM, Beaufort ID de (1992) Medical decisions concerning the end of life in children in The Netherlands. Am J Dis Child 146: 1429-1431

12. Younger S, Jackson DL, Allen M (1979) Staff attitudes towards the care of the critically ill in the medical intensive care unit. Crit Care Med 7:35-40 